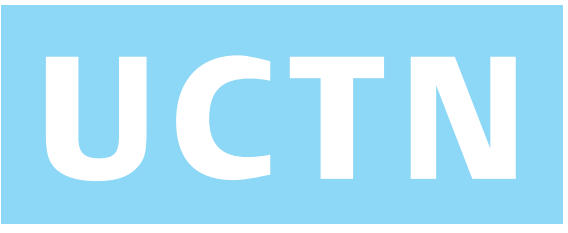

\title{
Epstein-Barr virus associated with a post- transplant lymphoproliferative disorder presenting as isolated gastrointestinal tract bleeding
}
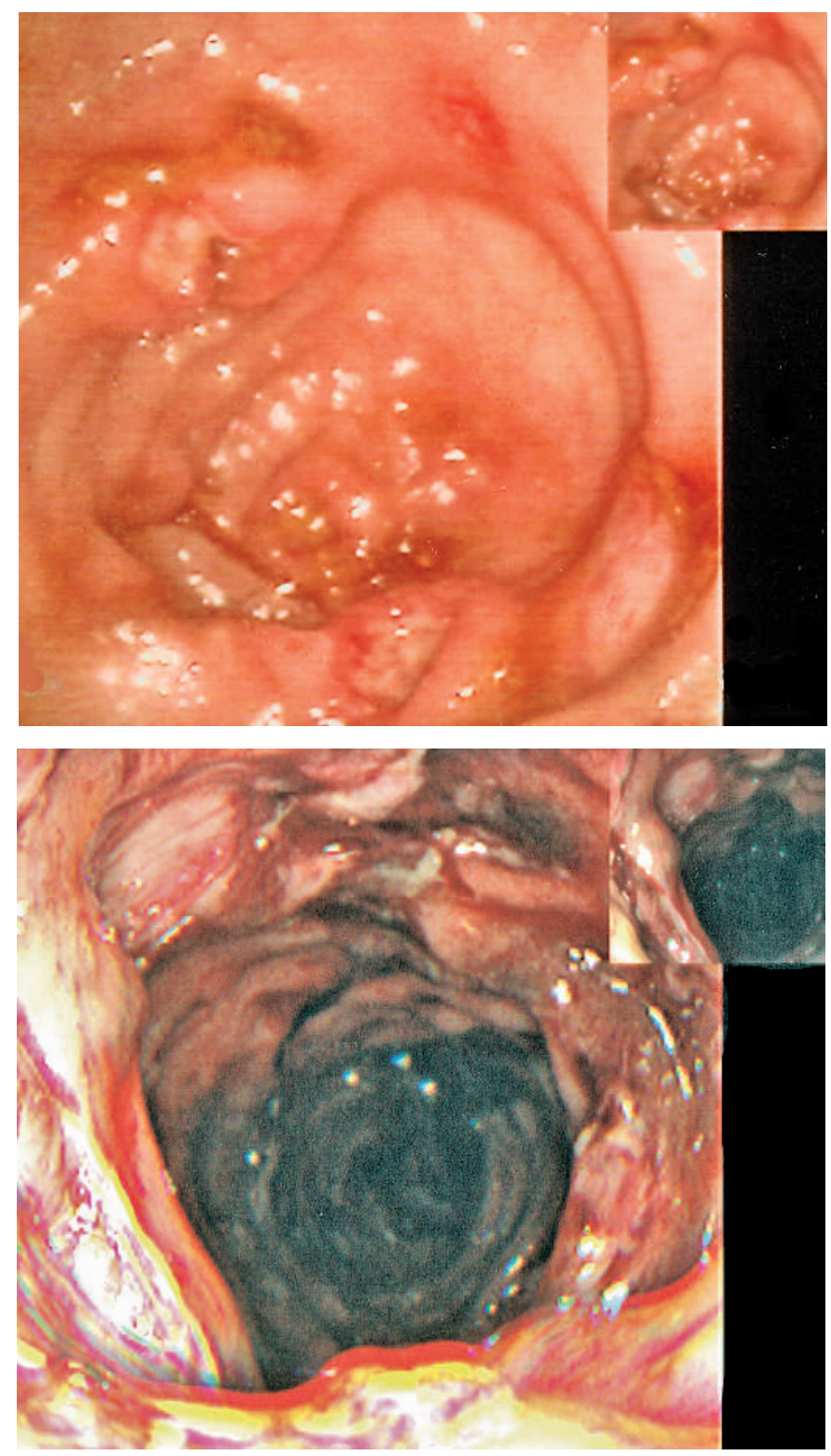

A 46-year-old woman underwent a cadaveric renal transplant. Three months later, she developed diarrhoea, fresh rectal bleeding, and cervical lymphadenopathy. Renal biopsy showed no evidence of rejection. Flexible sigmoidoscopy revealed multiple colonic ulcers, with no active bleeding (Figure 1). Biopsies showed post-transplant lymphoproliferative disorder (PTLD). The cells were positive for Epstein-Barr virus (EBV) latent membrane protein, and positive for CD20,
Figure 1 The descending colon on the initial endoscopy.

Figure 2 Findings at the second endoscopy.
CD23, Pax 5, and EBV DNA. Tacrolimus (Astellaf Pharma, Ireland) was discontinued and anti-CD20 antibody treatment (rituximab; Roche, Basle, Switzerland) was started.

However, 3 weeks later, another episode of rectal bleeding occurred. Flexible sigmoidoscopy showed necrotic, sloughy mucosa, with bleeding from multiple colonic ulcers (Figure 2). An emergency laparotomy revealed numerous vasculitic areas on serosal surfaces in the small and large bowel, corresponding to sites of bowel ulceration. Subtotal colectomy with end ileostomy was carried out. Histopathological analysis of the specimen confirmed EBV-associated PTLD. Despite continued anti-CD20 therapy, and inotropic/ventilatory support in the intensivecare unit, the patient died 14 days postoperatively.

PTLD is a complication of immunosuppression following organ transplantation. Eighty percent of cases are associated with EBV [1]. Following renal transplantation in adults, the incidence of PTLD is $0.5-2.0 \%$ [2]. The time of onset depends on the organ transplanted and on the type and duration of immunosuppression; with tacrolimus, the mean is 4 months [2]. Gastrointestinal tract involvement occurs in up to $20 \%$ of cases of PTLD, due to the presence of mucosaassociated lymphoid tissue [3]. The distal small bowel is most commonly affected [4]. Reducing immunosuppression (the risk of graft rejection should be considered), if successful, usually results in improvement within 2-4 weeks. If this fails, a high mortality rate of $50-90 \%$ is reported [1]. Anti-B-cell antibodies can be used.

In conclusion, rectal bleeding and isolated gastrointestinal PTLD, as in this case, are uncommon [5], but should be considered in post-transplant patients. The condition can be aggressive and fatal despite treatment. Early endoscopy with biopsies for even mild nonspecific symptoms of PTLD is therefore advised.

Endoscopy_UCTN_Code_CCL_1AD_2AZ

Endoscopy_UCTN_Code_CCL_1AD_2AJ

DOI: $10.1055 / \mathrm{s}-2006-944922$ 
K. Badham ${ }^{1}$, A. Mirchandani², N. Arumainayagam ${ }^{1}$, D. R. West ${ }^{2}$

${ }^{1}$ Dept. of General Surgery, Southmead Hospital, Bristol, United Kingdom

${ }^{2}$ Dept. of General Surgery, Weston General Hospital, Weston-super-Mare, United Kingdom.
References

${ }^{1}$ Taylor AL, Marcus R, Bradley JA. Post-transplant lymphoproliferative disorders (PTLD) after solid organ transplantation. Crit Rev Oncol Hematol 2005; 56: 155-167

${ }^{2}$ Boubenider S, Hiesse C, Goupy C et al. Incidence and consequences of post-transplant lymphoproliferative disorders. J Nephrol 1997; 10: 136- 145

${ }^{3}$ Hachem RR, Chakinala MM, Yusem RD et al. Abdomino-pelvic lymphoproliferative disease after lung transplantation: presentation and outcome. Transplantation 2004; 77: $431-437$

${ }^{4}$ Green M. Management of Epstein-Barr virus-induced post-transplant lymphoproliferative disease in recipients of solid organ transplantation. Am J Transplant 2001; 1: $103-108$

${ }^{5}$ Sebire NJ, Malone M, Risdon RA, Ramsay AD. Epstein-Barr virus-associated lymphoproliferative disorder presenting as apparently isolated gastrointestinal lesions in childhood. Pediatr Dev Pathol 2005; 8: 88-91
Corresponding author

A. Mirchandani, MB Ch. B. MRCS

Department of General Surgery Weston General Hospital

Weston-super-Mare BS23 4TQ

United Kingdom

Fax: $\quad$ +44-1934-636363

Email: anjaly@doctors.org.uk 